\title{
Prevalencia y perfil de susceptibilidad antimicrobiana en bacterias aisladas de úlceras crónicas infectadas en adultos
}

\author{
Víctor Silva, Alicia Marcoleta, Viviana Silva, Dángelo Flores, \\ Teresa Aparicio, Isabel Aburto, Cecilia Latrach y Naldy Febré
}

\section{Prevalence and susceptibility pattern of bacteria isolated from infected chronic wounds in adult patients}

Background: Chronic wounds are considered a public health problem that may be complicated by bacterial infections, mainly caused by resistant strains. Aim: To study the bacteria prevalence and antimicrobial susceptibility in samples from adult patients with chronic wounds. Methods: Patients treated at National Institute of Wounds Foundation between May and July 2014, with chronic ulcers in lower extremities with clinical inflammatory signs were recluted. Samples were cultured in aerobic and anaerobic atmosphere and species identification was performed by API (Biomerieux) galleries. The in vitro susceptibility was evaluated according to the Kirby Bauer method. Results: From 73 patients, 46 had infected wounds most of them were venous ulcers (33) with prevalence in polymicrobial infections and 10 with foot-diabetes ulcers with prevalence in monomicrobial infections $(\mathrm{p} \leq 0.05)$. Sixty-eight strains were isolated and Enterobacteriaceae were predominant in monomicrobial infection $(\mathrm{p} \leq 0.05)$ and the other groups were slightly higher in polymicrobial infection. The main species were Staphylococcus aureus (24\%) followed by P. aeruginosa (18\%). Fifty strains (77\%) were resistant or multi-resistance. We emphasize resistance of $S$. aureus to ciprofloxacin (50\%) and cefoxitin (37.5\%), thus identifying resistance to methicillin in the community (CA-SAMR), all of which are sensitive to cotrimoxazole. Enterobacteria showed sensitivity to amikacin (95.5\%), P. aeruginosa showed resistance to ciprofloxacin (33.3\%) with high sensitivity to gentamicin (91.7\%) and amikacin (83.3\%), while Acinetobacter spp showed resistance to ciprofloxacin and ceftazidime in $60 \%$, with $100 \%$ sensitivity to imipenem. 50\% Streptococcus $\beta$ hemolytic showed resistance to clindamycin and penicillin. Conclusion: These data provide epidemiological information on chronic wound infections, representing support for diagnosis, treatment and management of this pathology.

Key words: Chronic wounds, epidemiology, antibacterial resistance.

Palabras clave: Heridas crónicas, epidemiología, resistencia antimicrobiana.

\section{Introducción}

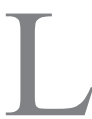

as úlceras o heridas crónicas se caracterizan por presentar falta de restauración íntegra en la forma anatómica del tejido lesionado y de su función ${ }^{1-3}$. Factores como la hipoxia, necrosis, exudación e infección, prolongan una o más etapas propias de la cicatrización, como la hemostasia, la inflamación, la proliferación y/o la remodelación ${ }^{4}$. Esta lesión se asocia a factores endógenos como enfermedades vasculares, hipertensión venosa y enfermedades metabólicas como diabetes mellitus, generando úlceras de extremidad inferiores que se clasifican según la patología de base ${ }^{1,4-6}$.

Como referencia, en el Reino Unido, la tasa de úlceras crónicas es de 1,48 por 1.000 habitantes, siendo más frecuente en mayores de 65 años (3,6\% de dicha población $)^{7,8}$. A nivel global se estima que la prevalencia de úlceras crónicas oscila en aproximadamente 1\% de la población general ${ }^{6-9}$. En Chile, aproximadamente 170.000 pacientes poseen algún tipo de herida o úlcera y el manejo está dirigido principalmente a las úlceras venosas, úlceras de pie diabético, úlceras hipertensivas y úlceras por presión ${ }^{6}$.

La úlcera venosa representa entre 75 y $80 \%$ del total de las úlceras vasculares, con una prevalencia de 0,5 a $0,8 \%$ y una incidencia de 2 a 5 casos nuevos por mil habitantes al año en países desarrollados, implicando pérdidas cercanas a los 2 millones de días laborales con costo cercano a los 3 billones de dólares ${ }^{7,9}$. En Chile, el gasto país asociado a úlceras venosas supera los $\$ 71$ millones de pesos ${ }^{10}$.

En países desarrollados, la ulceración del pie afecta a $15-25 \%$ de los pacientes con diabetes mellitus, siendo la primera causa de hospitalización asociada a dicha patología y precede hasta $85 \%$ de las amputaciones
Universidad Mayor, Santiago,

Chile.

Facultad de Ciencias,

Escuela de Tecnología Médica

(VS, AM, VS, DF, TA)

Escuela de Enfermería (CL).

Fundación Instituto Nacional de Heridas (FINH) (IA).

Universidad Andrés Bello.

Facultad de Enfermería (NF).

Los autores declaran no tener conflictos de interés.

Trabajo financiado con aportes de Conicyt: Proyecto FONDEF CA13110225.

Recibido (última versión): 21 de diciembre de 2017 Aceptado: 7 de febrero de 2018

Correspondencia a: Víctor Silva Vargas victor.silva@umayor.cl 
en este grupo. La tasa de re-ulceración a los 5 años es aproximadamente de $70 \%$, mientras que la infección en estas lesiones alcanza cifras de hasta $34 \%^{11-13}$. Úlceras hipertensivas se pueden observar con una prevalencia de $60 \%$ en pacientes diabéticos, con una media de 50 a 60 años y mayor incidencia en mujeres ${ }^{14}$.

La carencia de atención oportuna repercute en períodos de incapacidad laboral más prolongados, tratamientos costosos, hospitalizaciones reiteradas y cirugías que pueden llegar a amputación, invalidez e incluso evolucionar a óbito ${ }^{15,16}$.

Las úlceras crónicas no siempre manifiestan los síntomas clásicos de infección, como dolor, eritema, edema, calor y purulencia. Por tal motivo, se han propuesto otros signos específicos como exudado seroso, inflamación concomitante, retraso en la cicatrización, decoloración del tejido, tejido de granulación friable, mal olor y dehiscencia de la herida. A su vez, se ha empleado como indicador, la carga bacteriana y especie del patógeno identificado ${ }^{4,8,17,18}$.

Cuando la infección es superficial, generalmente se relaciona a un único agente patógeno, teniendo mejor pronóstico. En cambio, si la herida es profunda la infección puede ser producida por dos o más patógenos, la que se asocia con mayor riesgo de amputación ${ }^{8,13,15,19}$

Estudios han demostrado que las cepas que afectan frecuentemente las úlceras del pie diabético incluyen microorganismos aeróbicos y anaeróbicos sugiriendo que la mezcla de especies puede repercutir en la virulencia de las distintas especies bacterianas ${ }^{20,21}$. El rol patogénico de algunos microorganismos no está del todo claro, como es el caso de Enterococcus spp., Staphylococcus epidermidis, Streptococcus del grupo viridans y Corynebacterium spp., ya que, si bien han sido descritos como colonizadores, en asociación a otros microorganismos serían capaces de liderar el proceso infeccioso ${ }^{8,14,22-24}$.

Las úlceras crónicas infectadas se caracterizan por presentar etiología polimicrobiana, por ende, el uso de antimicrobianos de amplio espectro es el tratamiento recomendado ${ }^{8,9,15}$. Muchas bacterias pueden persistir en estas lesiones formando bio-películas, estructura que protege a las bacterias frente a la fagocitosis y la acción de los antimicrobianos, dificultando su erradicación ${ }^{18,22}$. La prescripción y administración inadecuada de antimicrobianos genera selección de cepas bacterianas resistentes lo que se asocia a mayor probabilidad de mortalidad cuando se compara con infecciones de heridas por bacterias sensibles $^{8,25,26}$.

Para el éxito del tratamiento en las úlceras crónicas infectadas es primordial la comprensión de la dinámica microbiana, tanto en su prevalencia como en sus patrones de susceptibilidad, lo cual irá en directo beneficio para apoyar la toma de decisiones en el manejo y tratamiento de la lesión.

Dado estos antecedentes, el presente estudio busca determinar el tipo de infección, prevalencia y patrón de susceptibilidad-resistencia antimicrobiana de especies bacterianas aisladas desde úlceras crónicas de pacientes atendidos en la Fundación Instituto Nacional de Heridas en Santiago de Chile.

\section{Material y Métodos}

El presente estudio fue de tipo descriptivo prospectivo, longitudinal y analítico, efectuado entre los meses de mayo y julio de 2014

\section{Pacientes y criterio de infección}

Se reclutaron pacientes con sospecha de heridas crónicas infectadas, previa obtención del consentimiento informado, autorizado por Comité de Ética de la Universidad Mayor. Los pacientes fueron atendidos en la Fundación Instituto Nacional de Heridas (FINH), que por convenio con el Ministerio de Salud (MINSAL), fueron derivados desde establecimientos de Atención Primaria del Sistema Público de la Región Metropolitana.

El tamaño de la muestra se obtuvo en base a la prevalencia de heridas o úlceras en la población adulta en Chile equivalente al $1 \%$, de 8 millones de adultos, con nivel de confianza de $99 \%$, error de muestreo 3\%. Se utilizó como fuente de datos la encuesta epidemiológica del Ministerio de Salud, año $2000^{13}$, considerándose para este estudio tres tipos de heridas crónicas: úlceras venosas, pie diabético y úlceras hipertensivas ${ }^{10,13}$.

\section{Definiciones}

Se consideró infección la presencia de uno o más signos y síntomas, de procesos infecciosos de la herida (dolor, eritema, edema, calor y purulencia) con presencia regular a abundante de agentes bacterianos (cocáceas y/o bacilos), acompañados de células epiteliales y leucocitos polimorfonucleares.

La muestra fue considerada como colonizada por microbiota si presentaba desarrollo bacteriano en el cultivo, sin acompañarse de leucocitos polimorfonucleares en la tinción de Gram y escasa o muy escasa presencia bacteriana.

\section{Procesamiento de la muestra}

La muestra clínica se recolectó por raspado con cureta y el material se traspasó a una tórula la que fue depositada en un tubo con caldo tioglicolato para su transporte a temperatura de $4{ }^{\circ} \mathrm{C}$ desde la FINH al Laboratorio de Investigación Microbiológica de la U. Mayor, donde se realizó procedimiento diagnóstico estándar, iniciado por una tinción de Gram con observación y análisis semicuantitativo, expresando los resultados como; muy escasa cantidad (+/-), escasa cantidad $(+)$, regular cantidad $(++)$ y abundante cantidad $(+++)$. 
Las muestras se sembraron en placas de cultivo con agar sangre de cordero, agar chocolate y agar MacConkey, incubándose en aerobiosis a $35-37{ }^{\circ} \mathrm{C}$ por $24 \mathrm{~h}$. Para el estudio de úlceras de pie diabético, se adicionó un cultivo en anaerobiosis empleando placas de agar Schaedler, incubándose en Jarra Gas-Pak, con un sistema generador de anaerobiosis a $35-37^{\circ} \mathrm{C}$, durante 48 a $72 \mathrm{~h}^{1,25}$.

Las cepas bacterianas fueron identificadas analizando las características macro y micromorfológicas, pruebas fisiológicas, enzimáticas y/o bioquímicas de los distintos microorganismos con ayuda de sistemas de galerías API $\left(\right.$ Biomerieux $\left.^{\circledR}\right)$ según tipo de agente bacteriano, con lectura visual y análisis en sofware API-Web versión $4.0\left(\right.$ Biomerieux $\left.^{\circledR}\right)$.

Para identificar crecimiento anaeróbico, las colonias se analizaron en microscopio estereoscópico, tinción de Gram de las colonias sospechosas y repique en dos placas de agar Schaedler, incubando una en anaerobiosis y otra en $\mathrm{CO}_{2}$. Para identificar la cepa anaerobia se contó con apoyo de la galería comercial API 20A (Biomerieux ${ }^{\circledR}$ ).

La susceptibilidad antimicrobiana fue evaluada mediante el método de difusión en agar (Kirby Bauer) en medio Müller Hinton, siguiendo las directrices establecidas por el Committee for Clinical Laboratory Standards Institute (CLSI) documento M02-A1127. En resumen, se preparó un inóculo 0,5 McFarland (1-2 x $\left.10^{8} \mathrm{UFC} / \mathrm{ml}\right)$, determinándose la $\mathrm{DO}_{625} \mathrm{~nm}(0,08 \mathrm{y}$ 0,13 unidades de Densidad Óptica). Luego, se sembró el medio Müller-Hinton utilizando una tórula embebida en el inóculo mediante la técnica de siembra en alfombra. Posteriormente, con la ayuda de un dispensador se depositaron los sensidiscos seleccionados para cada especie bacteriana. Luego, la placa se incubó $35-37^{\circ} \mathrm{C}$ por 18 a $24 \mathrm{~h}$ en aerobiosis. Se determinó el halo de inhibición de crecimiento en milímetros $(\mathrm{mm})$ y se registró el resultado con su respectiva interpretación como sensible, intermedio o resistente, según el CLSI M100-S23 ${ }^{28}$. Para este estudio se definió como bacteria multi-resistente a aquella cepa resistente a tres o más antimicrobianos ${ }^{26}$. Los antimicrobianos utilizados para el estudio de susceptibilidad por cada grupo bacteriano fueron seleccionados siguiendo recomendación de la guía CLSI ${ }^{27,28}$ y de trabajos de la literatura científica ${ }^{9,12,25,30,31}$ los que son presentados en la Tabla 1.

\section{Control de calidad y análisis estadístico}

Para pruebas de identificación y ensayos de susceptibilidad antimicrobiana se utilizó como control de calidad las cepas ATCC (American Type Culture Collection) de las especies Staphylococcus aureus $\left(\mathrm{ATCC}^{\circledR} 25923^{\mathrm{TM}}\right.$ ) y Pseudomonas aeruginosa (ATCC ${ }^{\circledR} 27853^{\mathrm{TM}}$ ).

El análisis estadístico para tipo de infección y prevalencia bacteriana se realizó aplicando la prueba de ${ }^{2} \mathrm{de}$ Pearson con ayuda del software Microsoft Excel versión
Tabla 1. Listado de antimicrobianos utilizados según microorganismos, con sus respectivos halos de inhibición e interpretación de susceptibilidad

\begin{tabular}{|c|c|c|c|c|}
\hline \multirow[t]{2}{*}{ Microorganismos } & \multirow[t]{2}{*}{ Antibióticos } & \multicolumn{3}{|c|}{ Puntos de corte $/ \mathrm{mm}$} \\
\hline & & S & 1 & $\mathbf{R}$ \\
\hline \multirow[t]{5}{*}{ Staphylococcus aureus } & Cefoxitina & $\geq 22$ & - & $\leq 21$ \\
\hline & Ciprofloxacina & $\geq 21$ & $16-20$ & $\leq 15$ \\
\hline & Tetraciclina & $\geq 19$ & $15-18$ & $\leq 14$ \\
\hline & Cotrimoxazol & $\geq 16$ & $11-15$ & $\leq 10$ \\
\hline & Ampicilina/sulbactam & $\geq 15$ & $12-14$ & $\leq 11$ \\
\hline \multirow[t]{5}{*}{ Staphylococcus coagulasa negativa } & Cefoxitina & $\geq 25$ & - & $\leq 24$ \\
\hline & Ciprofloxacina & $\geq 21$ & $16-20$ & $\leq 15$ \\
\hline & Tetraciclina & $\geq 19$ & $15-18$ & $\leq 14$ \\
\hline & Cotrimoxazol & $\geq 16$ & $11-15$ & $\leq 10$ \\
\hline & Ampicilina/sulbactam & $\geq 15$ & $12-14$ & $\leq 11$ \\
\hline \multirow{3}{*}{ Streptococcus $\beta$ hemolíticos } & Penicilina & $\geq 24$ & - & - \\
\hline & Eritromicina & $\geq 21$ & $16-20$ & $\leq 15$ \\
\hline & Clindamicina & $\geq 19$ & $16-18$ & $\leq 15$ \\
\hline \multirow[t]{3}{*}{ Enterococcus spp. } & Ampicilina & $\geq 17$ & - & $\leq 16$ \\
\hline & Vancomicina & $\geq 17$ & $15-16$ & $\leq 14$ \\
\hline & Gentamicina & $\geq 10$ & 7-9 & $\leq 6$ \\
\hline \multirow[t]{5}{*}{ Enterobacterias } & Cefuroxima & $\geq 23$ & $15-22$ & $\leq 14$ \\
\hline & Ceftazidima & $\geq 21$ & $18-20$ & $\leq 17$ \\
\hline & Ciprofloxacina & $\geq 21$ & $16-20$ & $\leq 15$ \\
\hline & Gentamicina & $\geq 15$ & $13-14$ & $\leq 12$ \\
\hline & Amikacina & $\geq 17$ & $15-16$ & $\leq 14$ \\
\hline \multirow[t]{5}{*}{ Pseudomonas aeruginosa } & Gentamicina & $\geq 15$ & 13-14 & $\leq 12$ \\
\hline & Amikacina & $\geq 17$ & $15-16$ & $\leq 14$ \\
\hline & Imipenem & $\geq 19$ & $16-18$ & $\leq 15$ \\
\hline & Ceftazidima & $\geq 18$ & $15-17$ & $\leq 14$ \\
\hline & Ciprofloxacina & $\geq 21$ & $16-20$ & $\leq 15$ \\
\hline \multirow[t]{7}{*}{ Acinetobacter spp. } & Cotrimoxazol & $\geq 16$ & $11-15$ & $\leq 10$ \\
\hline & Gentamicina & $\geq 15$ & $13-14$ & $\leq 12$ \\
\hline & Amikacina & $\geq 17$ & $15-16$ & $\leq 14$ \\
\hline & Imipenem & $\geq 16$ & $14-15$ & $\leq 13$ \\
\hline & Ceftazidima & $\geq 18$ & $15-17$ & $\leq 14$ \\
\hline & Ciprofloxacina & $\geq 21$ & $16-20$ & $\leq 15$ \\
\hline & Ampicilina/sulbactam & $\geq 15$ & $12-14$ & $\leq 11$ \\
\hline
\end{tabular}

Otros bacilos gramnegativos no fermentadores como Pseudomonas alcalígenes, Pseudomonas fluorescens y Bordetella avium, no presentan puntos de corte de halo de inhibición en milímetros para método de difusión en agar.

2007 y el software SPSS versión 22 considerando un nivel de confianza de $95 \%(\alpha=0,05)$.

\section{Resultados}

Se reclutaron 73 pacientes de los cuales 46 presentaron úlceras infectadas. Veinticinco presentaron infección monomicrobiana y 21 polimicrobiana correspondiendo la mayoría de las lesiones a úlcera venosa (n: 33), seguidas de úlcera de pie diabético (n: 10) y úlcera hipertensiva 
(n: 3), siendo úlcera venosa más frecuente en infección polimicrobiana y úlcera de pie diabético en infección monomicrobiana $(\mathrm{p} \leq 0,05)$. Los tres casos de úlcera hipertensiva fueron monomicrobianas (Tabla 2).

De los 46 pacientes, nueve tenían menos de 60 años, 18 entre 60 a 70 años y 19 sobre 70 años de edad, con media de 66 años.

En los pacientes con úlceras infectadas se aislaron 68 cepas bacterianas. En infecciones monomicrobianas hubo un predominio significativo de enterobacterias $(p \leq 0,05)$;

Tabla 2. Distribución de los 46 pacientes con úlceras infectadas según tipo de úlcera e infección

\begin{tabular}{lccc}
\hline Tipo de úlcera & $\begin{array}{c}\text { Monomicrobiana } \\
\mathbf{n}\end{array}$ & $\begin{array}{c}\text { Polimicrobiana } \\
\mathbf{n}\end{array}$ & $\begin{array}{c}\text { Total } \\
\mathbf{n}(\%)\end{array}$ \\
U. venosa & 14 & 19 & $33(72 \%)$ \\
U. pie diabético & 8 & 2 & $10(22 \%)$ \\
U. hipertensiva & 3 & 0 & $3(6 \%)$ \\
Total & 25 & 21 & $46(100 \%)$ \\
\hline$p<0,05$ & & & \\
\hline
\end{tabular}

Tabla 3. Distribución porcentual de las 68 cepas aisladas según especie bacteriana y tipo de infección

\begin{tabular}{lccc}
\hline Especies bacterianas & $\begin{array}{c}\text { Monomicrobiana } \\
\text { (n: } \mathbf{2 5}=\mathbf{1 0 0} \%)\end{array}$ & $\begin{array}{c}\text { Polimicrobiana } \\
\text { (n: } \mathbf{4 3}=\mathbf{1 0 0} \%)\end{array}$ & $\begin{array}{c}\text { Total } \\
\text { (n: 68 = 100\%) }\end{array}$ \\
\hline Staphylococcus aureus & $12 \%$ & $30 \%$ & $24 \%$ \\
\hline Pseudomonas aeruginosa & $20 \%$ & $16 \%$ & $18 \%$ \\
\hline Escherichia coli & $8 \%$ & $7 \%$ & $7 \%$ \\
\hline Morganella morganii & $8 \%$ & $5 \%$ & $6 \%$ \\
\hline Proteus mirabilis & $8 \%$ & $2 \%$ & $4 \%$ \\
\hline Providencia rettgeri & $8 \%$ & $2 \%$ & $4 \%$ \\
\hline Acinetobacter lwofii & - & $7 \%$ & $4 \%$ \\
\hline Enterococcus faecalis & - & $7 \%$ & $4 \%$ \\
\hline Acinetobacter baumannii & $4 \%$ & $2 \%$ & $3 \%$ \\
\hline Klebsiella oxytoka & $8 \%$ & - & $3 \%$ \\
\hline Enterobacter sakazakii & $4 \%$ & $2 \%$ & $3 \%$ \\
\hline Enterobacter cloacae & - & $5 \%$ & $3 \%$ \\
\hline Staphylococcus xylosus & $8 \%$ & - & $3 \%$ \\
\hline Streptococcus agalactiae & - & $5 \%$ & $3 \%$ \\
\hline Serratia marcescens & $4 \%$ & - & $1 \%$ \\
\hline Pseudomonas alcaligenes & - & $2 \%$ & $1 \%$ \\
\hline Pseudomonas fluorescens & - & $2 \%$ & $1 \%$ \\
\hline Bordetella avium & - & $2 \%$ & $1 \%$ \\
\hline Staphylococcus schleiferi & $4 \%$ & - & $1 \%$ \\
\hline Streptococcus equi Ssp equi & $4 \%$ & - & $1 \%$ \\
\hline Streptococcus uberis & - & $2 \%$ & \\
\hline p > 0,05. & & & $1 \%$ \\
\hline
\end{tabular}

en cambio, se observó leve predominio de los demás grupos bacterianos en las infecciones polimicrobianas (Gráfico 1).

Entre las especies identificadas, predominó Staphylococcus aureus (24\%) principalmente en infección polimicrobiana, seguida de $P$. aeruginosa (18\%) la que fue más frecuente en infección monomicrobiana, sin alcanzar significancia estadística $(\mathrm{p}>0,05)$. Las demás especies identificadas tuvieron menor representatividad (Tabla 3 ).

En la Tabla 4, se muestra el patrón de susceptibilidad de 65 de las 68 cepas (tres cepas correspondieron a especies que no cuentan con puntos de corte definidos para su interpretación). En resumen, se observó sólo 15 $(23 \%)$ cepas sensibles a todos los antibacterianos estudiados, mientras 37 (57\%) fueron resistentes a uno o dos antibacterianos y $13(20 \%)$ presentaron multi-resistencia $(\mathrm{p} \leq 0,05)$. El patrón de susceptibilidad fue comparable entre cepas recuperadas de infecciones mono y polimicrobianas (Tabla 4).

En la Figura 1, destaca la alta sensibilidad de Staphylococcus coagulasa negativa, en especial a tetraciclina y ciprofloxacina, mientras $S$. aureus evidencia resistencia a ciprofloxacina (50\%) y cefoxitina $(37,5 \%)$ identificando cepas de $S$. aureus resistente a meticilina adquirido en la comunidad (SARM-AC), siendo todas sensibles a tetraciclina y cotrimoxazol. Streptococcus $\beta$ hemolítico presentó $50 \%$ de resistencia a clindamicina, eritromicina y penicilina. Enterococcus faecalis presentó $66,7 \%$ de resistencia a ciprofloxacina y $100 \%$ de sensibilidad a vancomicina y ampicilina. Se determinó resistencia de enterobacterias a cefuroxima $(36,4 \%)$ y alta sensibilidad a amikacina $(95,5 \%)$.

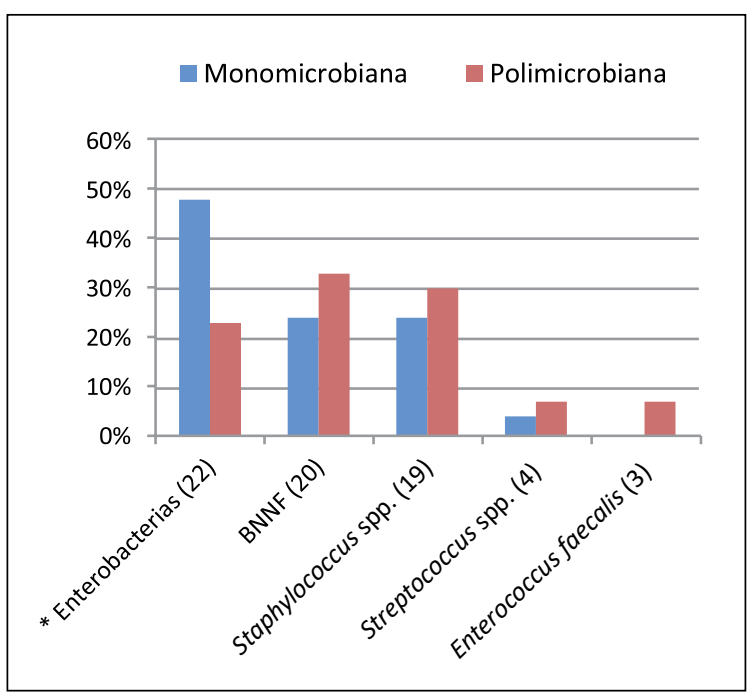

Gráfico 1. Distribución porcentual de las 68 cepas aisladas de infección monomicrobiana (25) y polimicrobiana (43) según grupo bacteriano. ${ }^{*} p=0,05$ (Enterobacterias comparadas con los demás grupos bacterianos). 


\begin{tabular}{|c|c|c|c|c|c|c|c|c|c|}
\hline \multirow[t]{2}{*}{ Grupo bacteriano/n } & \multicolumn{3}{|c|}{ Monomicrobiano ( $n=24$ cepas) } & \multicolumn{3}{|c|}{ Polimicrobiano ( $n=41$ cepas) } & \multicolumn{3}{|c|}{ Total ( $n=65$ cepas) } \\
\hline & $\mathbf{S}$ & $\mathbf{R}$ & MR & $\mathbf{S}$ & $\mathbf{R}$ & MR & $\mathbf{S}$ & $\mathbf{R}$ & MR \\
\hline Staphylococcus spp./19 & 2 & 4 & 0 & 4 & 9 & 0 & 6 & 13 & 0 \\
\hline Streptococcus spp./4 & 1 & 0 & 0 & 0 & 1 & 2 & 1 & 1 & 2 \\
\hline Enterococcus faecalis/3 & 0 & 0 & 0 & 0 & 3 & 0 & 0 & 3 & 0 \\
\hline Enterobacterias/22 & 4 & 5 & 3 & 0 & 7 & 3 & 4 & 12 & 6 \\
\hline BGNNF/17 & 0 & 3 & 2 & 4 & 5 & 3 & 4 & 8 & 5 \\
\hline Total & 7 & 12 & 5 & 8 & 25 & 8 & $15 *(23 \%)$ & $37 *(57 \%)$ & $13^{*}(20 \%)$ \\
\hline
\end{tabular}

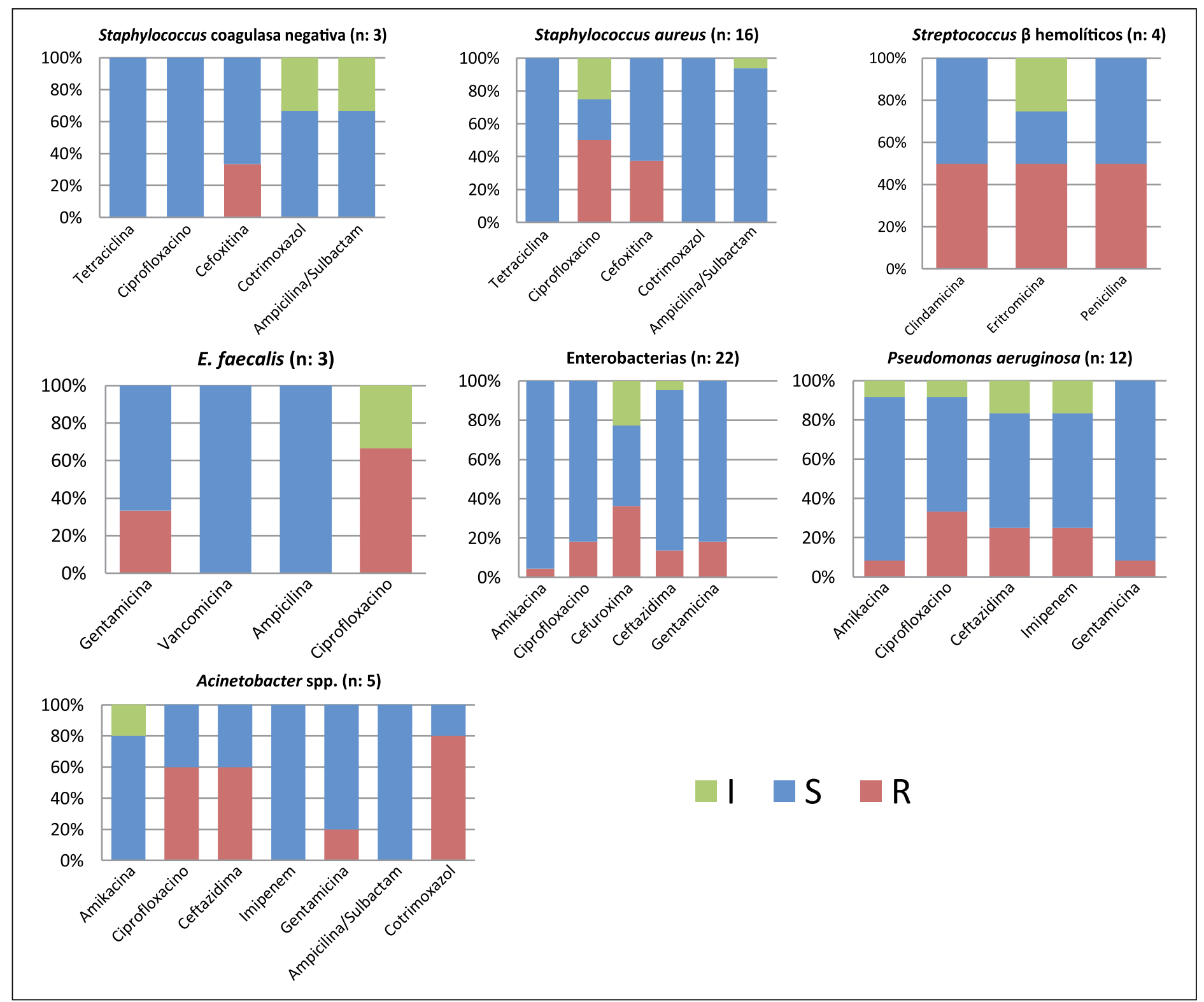

Figura 1. Perfil de susceptibilidad a cada antimicrobiano según grupo bacteriano. 
Entre los bacilos gramnegativos no fermentadores (BGNNF) destacamos a $P$. aeruginosa con resistencia a ciprofloxacina $(33,3 \%)$, alta sensibilidad a gentamicina $(91,7 \%)$ y amikacina $(83,3 \%)$, mientras Acinetobacter spp presentó resistencia a ciprofloxacina y ceftazidima en $60 \%$, con $100 \%$ de sensibilidad a imipenem y ampicilina/ sulbactam.

Pese a la definición inicial de efectuar cultivos anaerobios de cada muestra, el resultado fue infructuoso en todas las ocasiones.

\section{Discusión}

Se estima que las heridas crónicas se han incrementado debido a las patologías de base como enfermedades cardiovasculares y diabetes mellitus, entre otras ${ }^{1,5,16}$. Entre las afecciones más comunes en este tipo de úlceras están las infecciones que retrasan la cicatrización de la misma, principalmente cuando se presentan con microorganismos resistentes. Uno de los pilares en el tratamiento inicial corresponde al uso de antimicrobianos y para poder lograr un efecto positivo en el tratamiento utilizado, se debe conocer las características microbiológicas de la infección. Por lo tanto, para el éxito del tratamiento en este tipo de heridas es primordial estudiar la dinámica microbiana, tanto en su prevalencia como en sus patrones de susceptibilidad, lo que irá en directo beneficio para la remisión de la lesión y evolución del paciente pris, $^{8,19}$.

El presente estudio incorporó a 73 pacientes con úlceras, 46 de ellos evidenciaron infección de las lesiones. La alta frecuencia de infección de úlcera crónica diagnosticada clínica y microbiológicamente ha sido documentada por varios autores, indicando factores asociados a la enfermedad de base como una de las causas principales $^{1-2,4,16,23}$, así como su prevalencia en adultos mayores ${ }^{8,9}$, concordando con la distribución etaria de pacientes en este estudio. No obstante, esta serie discrepa de lo reportado en úlcera hipertensiva que afecta mayoritariamente a menores de 60 años ${ }^{14}$.

El tipo de herida infectada prevalente fue úlcera venosa, seguido de pie diabético, concordando con trabajos previos tanto nacionales como extranjeros ${ }^{6,11-13}$. En úlcera venosa, predominó la infección polimicrobiana, lo que concuerda con otros estudios ${ }^{8,19}$. En úlcera de pie diabético fue más frecuente el diagnóstico de infección monomicrobiana, siendo este resultado distinto a lo reportado anteriormente por otros autores como Citron y cols., en E.U.A. ${ }^{25}$. Estos autores obtuvieron las muestras, además de efectuar curetaje de las heridas, mediante biopsia de tejido o técnicas de aspiración en lugar de hisopo, lo que podría explicar la mayor frecuencia de infecciones polimicrobianas reportada por ellos.

Globalmente, se aislaron 68 cepas de úlceras infectadas con predominio significativo de enterobacterias en infecciones monomicrobianas, lo que difiere de estudios anteriores en los que predominaron las especies grampositivas ${ }^{4,8,15,19,23,25}$.

En las muestras de úlcera de pie diabético no se aislaron bacterias anaerobias, debido probablemente al medio empleado para su transporte, sumado al lapso de tiempo asociado a su traslado al laboratorio para su posterior procesamiento. En cambio, Citron y cols., sí describen el aislamiento de bacterias anaerobias en muestras de úlceras de pie diabético en 1,3\% mientras que el rendimiento global del cultivo anaerobio en conjunto con bacterias aerobias, alcanzó a 48\% $\%^{25}$.

Al analizar la frecuencia de especies, se apreció una gran diversidad de agentes aislados en heridas crónicas infectadas, identificando en total 21 especies bacterianas. Las predominantes fueron $S$. aureus y $P$. aeruginosa, mientras que E. faecalis y las especies de Streptococcus $\beta$ hemolíticos se aislaron en baja frecuencia, lo que concuerda con otros estudios ${ }^{8,24-25}$. Staphylococcus aureus presentó una frecuencia mayor en infecciones polimicrobianas. Por otro lado, las especies aisladas Staphyloccus xylosus y Staphylococcus schleiferi, si bien pueden formar parte de la microbiota fisiológica, algunos autores las han asociado como agentes de infecciones en úlceras crónicas, principalmente en procesos polimicrobianos ${ }^{4,24,25}$. Pseudomonas aeruginosa fue levemente más frecuente en infección monomicrobiana, siendo este resultado diferente a reportado por otros autores ${ }^{4,25}$. Las especies del género Streptococcus identificadas fueron $S$. uberis, $S$. equi subespecie equi y $S$. agalactiae, dato que difiere de Hefni y cols., quienes indican a Streptococcus del grupo A, específicamente $S$. pyogenes, como el agente asociado en infecciones de úlcera en pie diabético ${ }^{23}$.

El perfil de susceptibilidad in vitro de las cepas aisladas mostró frecuencia significativamente superior de cepas resistentes/multi-resistentes, concordando con varios estudios de países tanto desarrollados como en vías de serlo, lo que limita las opciones terapéuticas en los pacientes $^{8,19,24,25,31-33}$. Es importante mencionar que se reporta mayor mortalidad en pacientes con heridas crónicas infectadas por microorganismos resistentes que en pacientes con heridas crónicas infectadas con bacterias sensibles ${ }^{8,25}$.

Las enterobacterias presentaron mayor resistencia a cefuroxima y alta sensibilidad frente ceftazidima así como frente a ciprofloxacina, gentamicina y en especial frente amikacina ${ }^{31}$. Un centro clínico de Buenos Aires, Argentina, reporta que la resistencia a cefalosporinas está dada principalmente por $\beta$-lactamasas de espectro extendido (BLEE) (90\%), mientras que los mecanismos de hiperproducción de cefalosporinas tipo AmpC plasmídica y carbapenemasas de tipo KPC son detectados con menor frecuencia ${ }^{34}$.

En tanto, los BGNNF presentaron elevada resistencia a ciprofloxacina y ceftazidima; sin embargo, P. aerugi- 
nosa presentó alta sensibilidad a gentamicina $(91,7 \%)$ y amikacina (83,3\%), mientras Acinetobacter spp presentó $100 \%$ de sensibilidad a ampicilina/sulbactam e imipenem. En otras publicaciones se destaca la resistencia a ciprofloxacina con porcentajes entre 33 y $45 \%$ en BGNNF destacando resistencia de Acinetobacter spp., de hasta $50 \%$ a ceftazidima y a cotrimoxazol, en infecciones de úlceras crónicas ${ }^{8,19,24,31}$. En nuestro estudio, la resistencia fue mayor entre las cepas de BGNNF aisladas.

En Staphylococcus aureus, 37,5\% presentaron resistencia a cefoxitina, (antimicrobiano empleado para testear la resistencia in vitro a meticilina), dando a conocer la elevada presencia de cepas resistentes a meticilina adquiridas en la comunidad (SARM-AC) en úlceras crónicas infectadas de pacientes atendidos en forma ambulatoria, hallazgo que respalda la mayor frecuencia de este agente en América Latina comparado con Europa ${ }^{32,33}$. Esta frecuencia de SARM-AC es mayor a lo reportado en pacientes con úlcera de pie diabético en E.U.A. y menor a los casos de SARM aislados de heridas infectadas asociadas a atención de salud en ambiente hospitalario ${ }^{8}$. Los SARM$\mathrm{AC}$ aislados en nuestro estudio son sensibles in vitro frente a tetraciclinas y cotrimoxazol, lo que concuerda con otros estudios ${ }^{32,33}$. Apoyamos lo manifestado por algunos autores, quienes señalan que cepas de Staphylococcus spp con esta resistencia representan un problema que ha ido en aumento a nivel mundial, cuyas cifras reportadas son crecientes y preocupantes para la comunidad, avalando que SARM-AC es de fácil diseminación ${ }^{4,19,32,33}$.

En las cepas de Streptococcus $\beta$ hemolítico, se observó un patrón de resistencia a penicilina, eritromicina y clindamicina homogéneo y, de las tres cepas de E. faecalis, dos presentaron resistencia a ciprofloxacina mientras que todas respondieron a vancomicina y ampicilina, coincidiendo con datos reportados en otras latitudes como España ${ }^{35}$ e India $^{29}$, aunque difieren tanto en frecuencia como en patrón de susceptibilidad con datos provenientes de E.U.A. ${ }^{25}$.

Resaltamos lo primordial que significa realizar el estudio microbiológico desde la muestra representativa del sitio de infección de úlcera crónica, para definir si el cuadro es mono o polimicrobiano, más la identificación de las especies y su respectiva susceptibilidad antimicrobiana. Se genera así información vital que apoyará al equipo médico en la toma de decisiones con evidencia científica, facilitando la elección del antimicrobiano apropiado, complementado por el manejo de los procedimientos de curación, trabajo que debe ser interdisciplinario apoyando así resultados de otros autores $3,6,11,15,21,24,30$.

Es necesario conocer y comprender la dinámica microbiana en estas infecciones, tanto en el tipo de infección, prevalencia de especies y en sus patrones de susceptibilidad para entregar información relevante que apoye el manejo y tratamiento en los pacientes que padezcan este tipo de patologías.

Agradecimientos. A Andrea Báez por su ayuda en el análisis estadístico, a las Enfermeras y Técnicos de Enfermería de la FIHN, Técnicos de Laboratorio Estefanía Reilich y Maribel Cruces, así como a la Auxiliar Amelia Devia de la U. Mayor, por su apoyo contante en la preparación y manejo de insumos y materiales.

\section{Resumen}

Introducción: Las úlceras crónicas son un problema de salud pública, agravándose por infecciones bacterianas causadas principalmente por agentes resistentes. Objetivo: Estudiar prevalencia y perfil de susceptibilidad en bacterias aisladas de úlceras crónicas en pacientes adultos. Pacientes y Métodos: Pacientes atendidos en la Fundación Instituto Nacional de Heridas entre mayo y julio de 2014, con úlceras crónicas en extremidades inferiores con signos inflamatorios clínicos. Las muestras fueron cultivadas en aerobiosis y anaerobiosis y para la identificación bacteriana se empleó el sistema de galerías API (Biomerieux). La susceptibilidad in vitro se evaluó según el método de Kirby Bauer. Resultados: Se reclutaron 73 pacientes, entre quienes 46 presentaron úlceras infectadas, diagnosticándose 33 úlceras venosas con predominio de infección polimicrobiana y 10 úlceras de pie diabético con predominio de infección monomicrobiana ( $\mathrm{p} \leq 0,05)$. Se aislaron 68 cepas de los 46 pacientes con úlcera infectada. Las enterobacterias predominaron en infección monomicrobiana $(\mathrm{p} \leq 0,05)$ y los demás grupos bacterianos fueron levemente más frecuentes en infección polimicrobiana. La especie prevalente fue Staphylococcus aureus (24\%) seguida de Pseudomonas aeruginosa (18\%). Cincuenta cepas (77\%) presentaron resistencia a uno o más antibacterianos. Destacamos resistencia de $S$. aureus a ciprofloxacina $(50 \%)$ y cefoxitina $(37,5 \%)$ identificándose así resistencia a meticilina en la comunidad (SARM-AC), siendo todas sensibles a cotrimoxazol. Las enterobacterias presentaron resistencia a sensibilidad a amikacina (95,5\%), P. aeruginosa evidenció resistencia a ciprofloxacina $(33,3 \%)$ con alta sensibilidad a gentamicina (91,7\%) y amikacina (83,3\%), mientras Acinetobacter spp presentó resistencia a ciprofloxacina y ceftazidima en $60 \%$, con $100 \%$ de sensibilidad a imipenem. Streptococcus $\beta$ hemolítico presentó $50 \%$ de resistencia a clindamicina y penicilina. Conclusión: Estos datos entregan información epidemiológica de infecciones de úlceras crónicas, representando un apoyo al diagnóstico, tratamiento y manejo de esta patología. 


\section{Referencias bibliográficas}

1.- Saavedra Lozano J, Santos Sebastián M, González F, Hernández Sampayo M T, Navarro Gómez M L. Capítulo 17: Infecciones bacterianas de la piel y tejidos blandos. Junta Directiva de la Sociedad Española de Infectología Pediátrica editores. Protoccolos Diagnóstico-Terapéuticos de Infectología Pediátricas. $3^{\circ}$ edición, España, ERGON; 2011, p. $159-175$.

2.- Stroncek J D, Reichert W M. Chapter 1: Overview of wound healing in different tissue types. IN: Reichert W M editor. Indwelling Neural Implants: Strategies for contending with the in vivo environment. Boca Raton (FL): CRC Press/Taylor \& Francis, 2008. Disponible en: http://www.ncbi.nlm.nih.gov/books/NBK3938

3.- Andrades D P, Sepúlveda S, González J. Curación avanzada de heridas. Rev Chil Cirugía 2004; 56: 396-403.

4.- Ki V, Rotstein C. Bacterial skin and soft tissue infections in adults: A review of their epidemiology, pathogenesis, diagnosis, treatment and site of care. Can J Infect Dis Med Microbiol 2008; 19: 173-84.

5.- Velnar T, Bailey T, Smrkolj V. The wound healing process: an overview of the cellular and molecular mechanisms. J Int Med Res 2009; 37: 1528-42. DOI: $10.1177 / 147323000903700531$

6.- Aburto I, Morgado P. Gestión en manejo avanzado de heridas y úlceras en Chile. Rev Chil Heridas Ostomias 2010; 1: 3-13.

7.- Nelezen O, Bergqvist D, Lindhagen A, Hallbook T. Chronic leg ulcers: an underestimated problem in primary health care among elderly patients. J Epidemiol Comm Health 2005; 55: 143-9.

8.- Howell J R S, Wilson M J, Hill K E, Howard A J, Price P E, Thomas D W. A review of the microbiology, antibiotic usage and resistance in chronic skin wounds. J Antimicrob Chemother 2005; 55: 143-9. DOI: $10.1093 / \mathrm{jac} / \mathrm{dkh} 513$

9.- Beebe-Dimmer J, Pfeifer J, Engle J, Schottenfeld D. The epidemiology of chronic venous insufficiency and varicose veins. Ann Epidemiol 2005; 15: 175-84. DOI: 10.1016/j. annepidem.2004.05.015.

10.- Campo C S. Manejo actual de la úlcera venosa. Rev Chil Heridas Ostomias 2010; 1: 31-9.

11.- Schast V B. Pie diabético: Introducción a su manejo. Rev Chil Heridas Ostomias 2010; 1: 14-22.

12.- Ministerio de Salud. Gobierno de Chile. Encuesta Nacional de Salud ENS 2009-2010 (Consultado el 24 de marzo de 2016), disponible en (http://www.minsal.gob.cl/portal/url/item/ bcb03d7bc28b64dfe040010165012d23.pdf)

13.- Calderón A. Perfil de pacientes en curación avanzada de ulceras de pie diabético, en el consultorio Pudahuel poniente de la Región Metropolitana de Santiago. Rev Chil Heridas Ostomias 2011; 2: 78-82.

14.- Galleri G, D'Angelo J, Morales M. Úlcera de Martorell. Fleb Linfo/Lect Vascu 2009; 12: 737-42.

15.- Bowler P G, Duerden B I, Armstrong D G. Wound microbiology and associated approaches to wound management. Clin Microbiol Rev 2001; 14: 244-69.

16.- Gardner S E, Frantz R A, Doebbeling B N. The validity of the clinical signs and symptoms used to identify localized chronic wound infection. Wound Repair Regen 2001; 9 (3): 78-86.

17.- Walker M, Metcalf D, Parsons D, Bowler P. A real-life clinical evaluation of a next-generation antimicrobial dressing on acute and chronic wound. J Wound Care 2015; 24: 11-22. doi: 10.12968/jowc.2015.24.1.11

18.- Metcalf D G, Bowler P G, Hurlow J. A clinical algorithm for wound biofilm identification. Acta Med Croatica 2016; 70: 73-9. DOI: 10.12968/ jowc.2014.23.3.137.

19.- Beltrán C, Fernández A, Gglio S, Biagini L, Morales R, Pérez J, et al. Tratamiento de la infección en el pie diabético. Rev Chilena Infect 2001; 18: 212-24.

20.- Thomas S, McCubbin P. An in vitro analysis of the antimicrobial properties of 10 silvercontaining dressings. J Wound Care 2003; 12 : 305-8.

21.- Aburto I, Juliet C, Salas C. El efecto de la polimihexanida con betaína en el biolfilm en las úlceras venosas. 2010. (Consultado el 15 marzo de 2016). Disponible en (http://www.inheridas. cl/wp- content/uploads/Revision-ResumenProntosan.pdf).

22.- Gastón Cartagena P. Biopelículas y heridas: desde la teoría hasta la práctica clínica. Rev Chil Heridas Ostomias 2010; 1: 47-56.

23.- Hefni H, Ibrahim M R, Attia K M, Moawad M M, El-Ramah A F, Shahin M M, et al. Bacteriological study of diabetic foot infection in Egypt. J Arab Soc Med 2012; 8: 26-32.

a. DOI: 10.7123/01.JASMR.0000429086.88718. bb.

24.- Thanni L O, Osinupebi O A, Deji-Agboola M. Prevalence of bacterial pathogens in infected wounds in a tertiary hospital, 1995-2001: any change in trend? J Natl Med Assoc 2003; 95: 1189-95.

25.- Citron D M, Goldstein E J, Merriam C V, Lipsky B A, Abramson M A. Bacteriology of moderate-to-severe diabetic foot infections and in vitro activity of antimicrobial agents. J Clin
Microbiol 2007; 45: 2819- 28. doi: 10.1128/ JCM.00551-07

26.- Bowler P G, Welsby S, Towers V, Booth R, Hogarth A, Rowlands V, et al. Multidrugresistant organism, wounds and topical antimicrobial protection. Int Wound $\mathrm{J}$ 2012; 9: 387-96. doi: 10.1111/j.1742481X.2012.00991.x. Epub 2012 May 29.

27.- Clinical and Laboratory Standards Institute (CLSI). Performance standards for antimicrobial disk susceptibility test; aproved standard M02-A11. Eleventh edition. Wayne, PA: Pensylvania, USA; 2012.

28.- Clinical and Laboratory Standards Institute (CLSI). Performance standards for antimicrobial susceptibility testing: TwentyThird informational supplement. Document M100-S23. Wayne, PA: Pensilvania, USA; 2013.

29.- Rajkumari N, Mathur P, Misra M C. Soft tissue and wound infections due to Enterococcus spp. among hospitalized trauma patients in a developing country. J Glob Infect Dis 2014; 6: 189-93.

30.- Auwaeter PG. Staphylococcus aureus. Johns Hopkins ABX Guide, 2016. (https:// www.hopkinsguides.com/hopkins/view/ Johns_Hopkins_ABX_Guide/540518/all/ Staphylococcus_aureus).

31.- Tapia A G P, Vázquez M S, Mata D C B, Mendosa R C, Morales L E F, Río L T V del, Romo S L. Prevalencia de infección de herida quirúrgica, causas y resistencia a los fármacos en el Hospital General de Zona núm. 2 del IMSS, San Luis Potosí. Rev Espec Méd-Quir 2012; 17: 261-5.

32.- Gabillot-Carre M, Roujeau J C. Acute bacterial skin infections and cellulitis. Curr Opin Infect Dis 2007; 20: 118-23.

33.- Cercenado E, Cuevas O, Marín M, Bouza E, Trincado P, Boquete T, et al. Communityacquired methicillin-resistant Staphylococcus aureus in Madrid, Spain: transcontinental importation and polyclonal emergence of Panton-Valentine leukocidin-positive isolates. Diagn Microbiol Infect Dis 2008; 61: 143-9. doi: 10.1016/j.diagmicrobio.2008.01.001. Epub 2008 Feb 15

34.- Nastro M, Montoto P, Saposnik E, García $\mathrm{S}$, Barberis C, Vay C, et al. Resistencia a cefaloposrinas de espectro extendido en enterobacterias sin AmpC inducible. Evaluación de los nuevos puntos de corte. Rev Argent Microbiol 2012; 44: 30-5.

35.- Cercenado E. Enterococcus: resistencias fenotípicas y genéticas y epidemiología en España. Enferm Infecc y Microbiol Clin 2011; 29 (Supl 5): 59-65. 